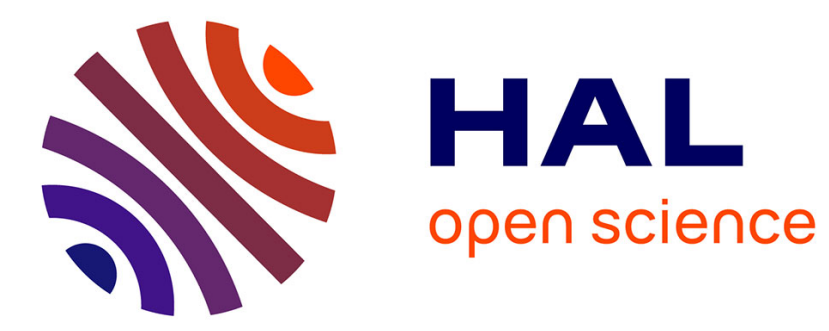

\title{
Raman cooling of spin-polarized cesium atoms in a crossed dipole trap
}

H Perrin, A Kuhn, I Bouchoule, T Pfau, C Salomon

\section{To cite this version:}

H Perrin, A Kuhn, I Bouchoule, T Pfau, C Salomon. Raman cooling of spin-polarized cesium atoms in a crossed dipole trap. EPL - Europhysics Letters, 1999, 46, pp.141 - 147. 10.1209/epl/i1999-00236-0 . hal-01076493

\section{HAL Id: hal-01076493 \\ https://hal.science/hal-01076493}

Submitted on 22 Oct 2014

HAL is a multi-disciplinary open access archive for the deposit and dissemination of scientific research documents, whether they are published or not. The documents may come from teaching and research institutions in France or abroad, or from public or private research centers.
L'archive ouverte pluridisciplinaire HAL, est destinée au dépôt et à la diffusion de documents scientifiques de niveau recherche, publiés ou non, émanant des établissements d'enseignement et de recherche français ou étrangers, des laboratoires publics ou privés. 


\title{
Raman cooling of spin-polarized cesium atoms in a crossed dipole trap
}

\author{
H. Perrin, A. Kuhn(*), I. Bouchoule, T. Pfau $(*)$, and C. Salomon \\ Laboratoire Kastler Brossel, École Normale Supérieure, \\ 24 rue Lhomond, 75231 Paris Cedex 05, France \\ (*)Present address: Fakultät für Physik der Universität Konstanz, Germany
}

(Dated: October 22, 2014)

\begin{abstract}
We describe a Raman cooling scheme that allows for the simultaneous polarization and cooling of cesium atoms. Using efficient chirped Raman pulses, cesium atoms confined in a red-detuned crossed dipole trap are $80 \%$ polarized and cooled to a temperature of $2.4 \mu \mathrm{K}$. After cooling, the phase-space density reaches $10^{-3}$. We have strong evidence that this value is limited by multiple photon scattering within the atomic cloud.
\end{abstract}

PACS numbers: $32.80 \mathrm{Pj}, 32.80 \mathrm{Qk}$

Since the first observation of Bose-Einstein condensation in 1995 using evaporative cooling in a magnetic trap [1 3], important efforts have been made to achieve quantum degeneracy by all optical methods [4, 5]. With current trap oscillation frequencies and atom number achievable with laser light, the onset of BEC requires temperatures below the single photon recoil temperature given by $k_{\mathrm{B}} T_{\text {rec }}=m v_{\text {rec }}^{2}=\hbar^{2} k^{2} / m$ were $k$ is the light wave number and $m$ the atomic mass. There are two laser cooling methods that can reach ultra-low temperatures when applied to free atoms, namely velocity-selective coherent population trapping (VSCPT) [6, 7] and Raman cooling [8 10]. Raman cooling of $\mathrm{Na}$ atoms confined in a dipole force trap has produced $T=0.42 T_{\text {rec }}$ and a phase-space density of $6.5 \times 10^{-3}$ [4]. Blue molasses 11] and Raman cooling [5] of Cs atoms confined in a dipole trap have produced phase-space densities of $10^{-3}$ and $2 \times 10^{-4}$, respectively. In these experiments, atoms were spread over all Zeeman substates. By contrast, gravitational Sisyphus cooling produced a sample of cold polarized rubidium atoms in a magnetic trap [12]. In this letter, we present a Raman cooling scheme that allows to simultaneously cool and polarize trapped cesium atoms in a weak bias magnetic field. Recently, a similar scheme was demonstrated on sodium atoms 13] and a temperature of $0.62 T_{\text {rec }}$ was obtained at a density of $4 \times 10^{11}$ atoms $/ \mathrm{cm}^{3}$. In our experiments, we accumulate more than $80 \%$ of the cesium atoms into the $\left|F=3, m_{F}=3\right\rangle$ stretched state at a temperature of $2.4 \mu \mathrm{K}$. The phase-space density reaches $10^{-3}$, a factor $7 \times 80 \%=5.6$ larger than in the unpolarized case. This figure represents a fourorder-of-magnitude gain over the phase-space density of a cesium magneto-optical trap (MOT) and a factor of 5 improvement over our previous results. We demonstrate that this value is limited by a heating mechanism associated with multiple photon scattering within the atomic cloud.

In our experiments, cesium atoms are collected for $1 \mathrm{~s}$ in a vapor cell MOT [14]. They are then loaded into a crossed dipole trap (fig. 1) at a peak density of about $5 \times 10^{11}$ atoms $/ \mathrm{cm}^{3}$. This trap consists of two $\mathrm{TEM}_{00}$
FIG. 1. Configuration of the trapping YAG beams and the cooling beams. A small magnetic field $(10 \mathrm{mG})$ along the vertical axis $\mathrm{z}$ provides our quantization axis. The YAG beams propagate in a vertical plane $x z$ and make an angle of $\alpha= \pm 53^{\circ}$ with the horizontal plane $x y$. The non degenerate eigenaxes of the resulting trap at the intersection of the beams are $x, y$ and $z$. The Raman beams propagate respectively along $x-y$ and $-z$ and are $\pi$ (resp. $\sigma^{-}$) polarized. The Raman transition is Doppler sensitive and induces a velocity change of $\sqrt{2} v_{\text {rec }}$ along the $\mathbf{x}-\mathbf{y}+\mathbf{z}$ direction. The repumping laser is present in two directions with polarizations $\sigma^{+}$or $\pi$.

Nd:YAG laser beams each having a power of $P_{Y A G}=5 \mathrm{~W}$ and which cross in their focal points where the waist is $w_{0}=100 \mu \mathrm{m}$ (see fig. 1). They propagate in the vertical $x z$-plane and make an angle of $\alpha= \pm 53^{\circ}$ with the horizontal axis $x$ (fig. 1a). The attractive potential is given by the ground-state light shift $\Delta E=\hbar \Omega_{\mathrm{YAG}}^{2}(\vec{r}) / 4 \Delta_{\mathrm{YAG}}$ where $\Omega_{\text {YAG }}$ is the Rabi frequency of the light field and $\Delta_{\mathrm{YAG}}$ is defined by $3 / \Delta_{\mathrm{YAG}}=1 / \Delta_{1}+2 / \Delta_{2}$. Here $\Delta_{i}$ is the detuning between the YAG laser frequency and the $D_{i}$ line of Cs. With $\Delta_{\mathrm{YAG}}=-2 \pi \times 64 \mathrm{THz}$, the photon scattering rate is less than $2 \mathrm{~s}^{-1}$. The lifetime of the trap is about one second, limited by collisions with the background gas. The polarizations of both beams are linear and parallel to each other, i.e. orthogonal to the $x z$-plane. An interference pattern in the vertical direction is therefore produced with a period of $665 \mathrm{~nm}$. In our previous work, we used this lattice to perform 1D Raman sideband cooling to the ground state [15, 16]. Here, by contrast, in order to produce a quasi-spherical trap, we use an acousto-optic modulator (AOM) to shift the frequency of one of the YAG beams by $100 \mathrm{MHz}$. The resulting interference pattern is moving at $33 \mathrm{~m} / \mathrm{s}$ and the cold atoms are not able to follow this fast motion of the lattice. They see a time-averaged potential without this fine spatial structure. Its depth $U_{\max } / k_{\mathrm{B}} \sim 70 \mu \mathrm{K}$ is given by the AC-Stark shift of either one of the YAG beams. This potential depth corresponds to oscillation frequencies $\nu_{x} \sim 170 \mathrm{~Hz}, \nu_{y}=5 / 4 \nu_{x} \sim 210$ 
FIG. 2. Principle of Raman cooling of polarized atoms. After each Raman transfer, the magnetic quantum number is increased by one. The repumping beam also contributes to polarize the atoms with its intense $\sigma^{+}$component. The $\pi$ component is necessary during cooling to pump the atoms in $|F=4, m=4\rangle$ back to $\mathrm{F}=3$.

$\mathrm{Hz}, \nu_{z}=3 / 4 \nu_{x} \sim 125 \mathrm{~Hz}$. Initially, a few $10^{5}$ atoms are transferred from the MOT to the crossed dipole trap. The $1 \sigma$ radius of the atomic cloud is $r=28 \pm 5 \mu \mathrm{m}$ and its temperature is $T_{0}=14 \pm 2 \mu \mathrm{K}$. This temperature is measured by an absorption imaging technique after a time of flight of $5 \mathrm{~ms}$ [1].

In order to polarize and cool the atoms, we repeat a cycle consisting of a stimulated Raman transition between the two hyperfine ground states changing the magnetic quantum number by +1 followed by a repumping pulse which brings the atoms back to the initial hyperfine state. A small magnetic field $(B=10 \mathrm{mG})$ along the vertical axis $z$ prevents from spin flips and provides a natural quantization axis. The Raman beams have the following configuration (fig. 1): the blue Raman beam propagates in the horizontal direction $\mathbf{x}-\mathbf{y}$ and is linearly polarized along $\mathrm{z}$ and the red Raman beam propagates along $-z$ and is $\sigma^{-}$polarized. After absorption of a $\pi$ photon in the blue Raman beam and stimulated emission of a $\sigma^{-}$ photon in the red Raman beam, an atom is transferred from $\left|6 S_{1 / 2}, F=3, m\right\rangle$ to $\left|6 S_{1 / 2}, F=4, m+1\right\rangle$ (fig. 2). Note that the Raman transfer is velocity selective with a Raman kick $\sqrt{2} v_{\text {rec }}$ ( $v_{\text {rec }}$ is the single photon recoil velocity, $3.5 \mathrm{~mm} / \mathrm{s}$ for cesium) along the $\mathbf{x}-\mathbf{y}+\mathbf{z}$ axis, which will be referred to as the Raman axis. This kick has a non-zero projection on each of the three eigenaxes of the trap, thus allowing Raman cooling in three dimensions. The detuning of the Raman beams from the $D_{2}$ line, $\Delta=-2 \pi \times 25 \mathrm{GHz}$, is much larger than the hyperfine structure of the excited state. Their frequency difference is $\Delta_{\text {HFS }}+\delta$ where $\delta$ is the Raman detuning and $\Delta_{\mathrm{HFS}}=2 \pi \times 9.2 \mathrm{GHz}$ is the splitting between the $\left|6 S_{1 / 2}, F=3\right\rangle$ and $\left|6 S_{1 / 2}, F=4\right\rangle$ hyperfine states of the cesium atom. The repumping laser is tuned to the $D_{2} F=4 \longrightarrow F^{\prime}=4$ transition and is split into two beams, a $\sigma^{+}$polarized saturating beam $(s=1)$ propagating along $-z$ and a much weaker $\pi$ polarized beam $\left(s=10^{-3}\right)$ propagating in the horizontal plane. The repumping process thus also contributes to polarize the atoms. This $\pi$-beam is not needed for polarizing the atoms but is necessary during Raman cooling for pumping the atoms out of the $|F=4, m=4\rangle$ state.

With this configuration of Raman beams, a Raman pulse is velocity selective and its effective Raman detuning depends on $m$ and the value of the bias field. To excite a broad velocity class with a transfer efficiency close to $100 \%$, the detuning of the Raman pulse is chirped [5]. For a given $m$, the chirp range determines the velocity
FIG. 3. Raman spectra before (dashed line) and after (full line) 15 repetitions of the polarizing sequence. The right peak corresponds to the $|F=3, m=3\rangle \longrightarrow|F=4, m=4\rangle$ transition. The Raman pulse is a Blackman pulse which satisfies the $\pi$ condition for the two extreme peaks. The population in $|F=3, m=3\rangle$ in this spectrum is $80 \%$.

classes which are adiabatically transferred to the other hyperfine state. First, we use the following sequence of Raman + repumping pulses to optically pump the atoms in the $\left|6 S_{1 / 2}, F=3, m=3\right\rangle$ state: With $B=10 \mathrm{mG}$, the detuning $\delta$ of a $400 \mu$ s Raman pulse is chirped from $+15 \mathrm{kHz}$ to $-44 \mathrm{kHz}$ to excite $|F=3, m=-3\rangle$ atoms with an initial velocity between $-5.3 v_{\text {rec }}$ and $+4.9 v_{\text {rec }}$ along the Raman axis. Atoms in $|F=3, m=+3\rangle$ are excited if their velocity is between $-12.5 v_{\text {rec }}$ and $-2.3 v_{\text {rec }}$. More generally, the Raman transfer from $\left|6 S_{1 / 2}, F=3, m\right\rangle$ to $\left|6 S_{1 / 2}, F=4, m+1\right\rangle$ for an atom with an initial velocity $(u-\sqrt{2} / 2) v_{\text {rec }}$ along the Raman axis occurs when $\delta=5.8 u+3.5 \times(2 m+1) \mathrm{kHz}$. This Raman pulse is followed by a $250 \mu$ s repumping pulse and the polarization sequence is repeated 15 to 20 times. In contrast to ref. [13], where a large bias magnetic field (430 $\mathrm{mG}$ ) was used to resolve the Doppler broadened Raman transitions of various $m_{F}$ substates, our method operates with a much weaker bias field allowing efficient simultaneous excitation of all $m_{F}$ states with a single chirped Raman pulse.

To measure the atomic polarization, we plot the number of atoms transferred from $F=3$ to $F=4$ by a Raman Blackman pulse [17] as a function of the Raman detuning $\delta$. This number is measured by the fluorescence light induced by a probe laser tuned to the $F=4 \rightarrow F^{\prime}=5$ transition at $852 \mathrm{~nm}$. This plot (a Raman spectrum) is produced with copropagating Raman beams. The red Raman beam is now aligned with the blue Raman beam and has a linear polarization orthogonal to $z$ (see fig. 1). The Raman transition is then Doppler insensitive and is only selective in internal energy. The resulting spectrum (fig. 3) consists of eight peaks, each peak corresponding to the two degenerate transitions $|F=3, m\rangle \longrightarrow|F=4, m+1\rangle$ and $|F=3, m+1\rangle \longrightarrow|F=4, m\rangle$ except for the extreme peaks which give the transfer efficiency from $|F=3, m=-3\rangle$ to $|F=4, m=-4\rangle$ and from $|F=3, m=3\rangle$ to $|F=4, m=4\rangle$. The population of the different magnetic sublevels is inferred from the relative height of the peaks. After 15 repetitions of the polarizing cycle, about $80 \%$ of the atoms are polarized in the $|F=3, m=3\rangle$ state, most of the others being in the $m=2$ state. The repumping process is responsible for a slight depolarization because the atoms may absorb several $\pi$ polarized photons before being pumped back to $F=3$. Depolarization may also occur because 
FIG. 4. Temperature in the crossed dipole trap as a function of the atom number, without cooling (open circles) or after $360 \mathrm{~ms}$ of Raman cooling (closed squares). The number of atoms and the temperature are inferred from a time-of-flight measurement after cooling. Note that the temperature does not depend on the atom number without Raman cooling.

of misalignment of the Raman beams with respect to the geometry imposed by the magnetic field. The final situation thus results from the equilibrium between polarizing and depolarizing processes [18].

Once the atoms are in the $|F=3, m=3\rangle$ state, Raman cooling is performed using the beam configuration of fig. 1. With respect to the quantization axis, the Raman beams couple the $|F=3, m=3\rangle$ state with the $|F=4, m=4\rangle$ state. The atoms with a velocity component opposite to the Raman axis and between 5.2 and $10.2 v_{\text {rec }}$ are excited using a $300 \mu$ s Blackman pulse chirped over $29 \mathrm{kHz}$ [5]. Because of the accumulation of the atoms in $m=3$, this pulse can be chosen more selective than the polarizing pulses. After each Raman transfer, the atoms are pumped back to the $|F=3, m=3\rangle$ state via the $\left|F^{\prime}=4, m^{\prime}=4\right\rangle$ excited state using a $400 \mu \mathrm{s}$ pulse. As above, the atoms may be depolarized during the repumping process by cycling between $F=4$ and $F^{\prime}=4$ before being pumped in $F=3$. However, due to the systematic change $\Delta m=+1$ at each Raman transfer, the polarization remains stable around $80 \%$ during Raman cooling. The trap temperature and atom number are measured by time-of-flight absorption imaging. After 500 cooling cycles (360 ms), a temperature of $2.4 \mu \mathrm{K}$ is obtained, corresponding to an rms velocity of $3.5 v_{\text {rec }}$. The number of atoms in the $|F=3, m=3\rangle$ ground state is $2.5 \times 10^{4}$. With a mean oscillation frequency of $170 \mathrm{~Hz}$, this corresponds to a peak density of $n_{0}=10^{12}$ atoms $/ \mathrm{cm}^{3}$ and a phase-space density of $10^{-3}$. This result is a four-order-of-magnitude improvement with respect to a magneto-optical trap. Thanks to the polarization of the atoms, the final phase-space density is higher than the one previously obtained with Raman cooling of unpolarized atoms in the trap with counter-propagating Raman beams [5]. However, the single-photon recoil temperature is not reached although our Raman cooling method for polarized atoms could in principle lead to subrecoil temperatures [4, 10, 13].

We now give evidence that the atomic temperature is likely to be limited by photon reabsorption during the repumping process: a spontaneous photon resonant with the $F=3 \longrightarrow F^{\prime}=4$ transition emitted during repumping can be scattered several times before it escapes the cloud, thus heating the atomic sample. This process becomes important at large values of the parameter $\beta=n_{0} \sigma r$, where $\sigma$ is the absorption cross section $\left(\sigma=6 \pi / k^{2}\right)[11,19]$. To illustrate this effect, we performed another set of experiments. We measured the temperature obtained after a given Raman cooling sequence for different values of the initial atom number $\mathrm{Ni}$ and hence different values of $\beta$. To vary $\beta$, we simply wait before starting cooling in the trap: the number of trapped atoms decreases with time due to collisions with the background gas. $\beta$ before cooling was varied between 2.8 and 1.1. The cooling sequence is the same as before. The final temperature deduced from time-of-flight measurement is plotted in fig. 4 as a function of the number of atoms $N_{f}$ at the end of the cooling process. Without cooling, the temperature does not depend on the atom number, except for a slight spontaneous evaporation for large values of $N$. On the contrary, the final temperature is an increasing function of $N_{f}$ as we apply the cooling sequence: we obtain $8 \mu \mathrm{K}$ for $5 \times 10^{4}$ atoms, whereas it decreases down to $4 \mu \mathrm{K}$ for $1.5 \times 10^{4}$ atoms. We have also observed that the cooling process induces an additional loss of atoms, typically $30 \%$ at $6 \mu \mathrm{K}$. We attribute this loss to hyperfine changing collisions 20, 21]. A detailed study of the heating rate as a function of $n_{0}$ and $r$ is beyond the scope of this paper. Additional evidence of the existence of reabsorption effects was obtained in another experiment with unpolarized atoms. It consisted of lowering the trapping potential, enforcing at the same time a slight evaporation of atoms 22]. A combination of Raman and evaporative cooling produced a temperature of $680 \mathrm{nK}\left(v_{\mathrm{rms}}=1.8 v_{\mathrm{rec}}\right)$ for a sample of $2 \times 10^{4}$ unpolarized atoms at a density of $4.3 \times 10^{11}$ at $/ \mathrm{cm}^{3}$ in the trap.

Cirac et al. and recently Castin et al. suggested to lower the repumping rate in order to limit the effect of photon reabsorption [23, 24]. If the two-atom model of ref. 24] is valid for a dense atomic cloud, we expect the reabsorption to be significantly reduced for repumping rates lower than the Doppler width $k v_{\text {rms }}$. Preliminary experimental results show a slight dependence of the final temperature with the pumping rate. The observed effect remains modest, the final temperature decreasing from 3 to $2.4 \mu \mathrm{K}$ as the pumping rate is lowered from $100 \mathrm{kHz}$ to $5 \mathrm{kHz}$ with the same cooling sequence. Note however that, in the case of cesium, because of the giant spin relaxation collisions of the $\left|F=m_{F}=4\right\rangle$ state 25] additional loss terms appear at very low repumping rates.

In conclusion, we have demonstrated a cooling scheme which prepares cesium atoms in a single internal state at very low temperatures. Using appropriate Raman and microwave pulses, it should be possible to transfer the atoms in any other magnetic state. Applications include the study of collisions in atomic fountains [26], atom optics experiments and pre-cooling for shortening the evaporation time in Bose-Einstein condensation.

$$
* * *
$$

We gratefully acknowledge Y. Castin for stimulating discussions. A.K. is indebted to the Alexander 
von Humboldt-Stiftung for support. T.P. was supported by the TMR network under the contract number FMRX-CT96-0002. This work was supported in part by CNES, NEDO and Collège de France. Laboratoire Kastler Brossel is Unité de recherche de l'Ecole Normale Supérieure et de l'Université Pierre et Marie Curie, associée au CNRS.

[1] M. Anderson, J. Ensher, M. Matthews, C. Wieman, and E. Cornell, Science 269, 198 (1995).

[2] K. Davis, M.-O. Mewes, M. Andrews, N. van Druten, D. Durfee, D. Kurn, and W. Ketterle, Phys. Rev. Lett. 75, 3969 (1995).

[3] C. Bradley, C. Sackett, J. Tollett, and R. Hulet, Phys. Rev. Lett. 75, 1687 (1995), erratum : ibid. 79 (6), 1170 (1997).

[4] H. J. Lee, C. S. Adams, M. Kasevich, and S. Chu, Phys. Rev. Lett. 76, 2658 (1996)

[5] A. Kuhn, H. Perrin, W. Hänsel, and C. Salomon, OSA TOPS on Ultracold Atoms and BEC (1996) 7, 58 (1997).

[6] A. Aspect, E. Arimondo, R. Kaiser, N. Vansteenkiste, and C. Cohen-Tannoudji, Phys. Rev. Lett. 61, 826 (1988).

[7] B. Saubaméa, T. W. Hijmans, S. Kulin, E. Rasel, E. Peik, M. Leduc, and C. Cohen-Tannoudji, Phys. Rev. Lett. 79, 3146 (1997)

[8] M. Kasevich and Phys. Rev. Lett. 69, 1741 (1992)

S. Chu,

[9] J. Reichel, O. Morice, G. M. Tino, and C. Salomon, EPL (Europhysics Letters) 28, 477 (1994).

[10] J. Reichel, F. Bardou, M. B. Dahan, E. Peik, S. Rand, C. Salomon, and C. Cohen-Tannoudji, Phys. Rev. Lett. 75, 4575 (1995)

[11] D. Boiron, A. Michaud, J. M. Fournier, L. Simard, M. Sprenger, G. Grynberg, and C. Salomon,
Phys. Rev. A 57, R4106 (1998)

[12] N. R. Newbury, C. J. Myatt, E. A. Cornell, and C. E. Wieman, Phys. Rev. Lett. 74, 2196 (1995).

[13] H. J. Lee and S. Chu, Phys. Rev. A 57, 2905 (1998).

[14] C. Monroe, W. Swann, H. Robinson, and C. Wieman, Phys. Rev. Lett. 65, 1571 (1990)

[15] H. Perrin, A. Kuhn, I. Bouchoule, and C. Salomon, Europhys. Lett. 42, 395 (1998).

[16] I. Bouchoule, H. Perrin, A. Kuhn, M. Morinaga, and C. Salomon, Phys. Rev. A 59, R8 (1999).

[17] M. Kasevich and S. Chu, Phys. Rev. Lett. 67, 181 (1991).

[18] We have also accumulated more than $95 \%$ of the atoms in the $|F=3, m=3\rangle$ state as follows: Using copropagating Raman beams we transfer atoms in the other $m$-states into $F=4$. Then, these atoms are pushed outside of the trap with a $\sigma^{+}$polarized resonant laser tuned to the cycling $|F=4\rangle \longrightarrow\left|F^{\prime}=5\right\rangle$ transition. However, when applying the cooling sequences we noticed that the polarization after cooling degraded to $80 \%$.

[19] D. Boiron, A. Michaud, P. Lemonde, Y. Castin, C. Salomon, S. Weyers, K. Szymaniec, L. Cognet, and A. Clairon, Phys. Rev. A 53, R3734 (1996).

[20] D. Sesko, T. Walker, C. Monroe, A. Gallagher, and C. Wieman, Phys. Rev. Lett. 63, 961 (1989)

[21] P. Lemonde, O. Morice, E. Peik, J. Reichel, H. Perrin, W. Hänsel, and C. Salomon, EPL (Europhysics Letters) 32, 555 (1995).

[22] C. S. Adams, H. J. Lee, N. Davidson, M. Kasevich, and S. Chu, Phys. Rev. Lett. 74, 3577 (1995)

[23] J. I. Cirac, M. Lewenstein, and P. Zoller, EPL (Europhysics Letters) 35, 647 (1996)

[24] Y. Castin, J. I. Cirac, and M. Lewenstein, Phys. Rev. Lett. 80, 5305 (1998).

[25] J. Söding, D. Guéry-Odelin, P. Desbiolles, G. Ferrari, and J. Dalibard, Phys. Rev. Lett. 80, 1869 (1998).

[26] K. Gibble and S. Chu, Phys. Rev. Lett. 70, 1771 (1993) 